\title{
A Rare Giant Midesophageal Diverticulum: Fever is the Only Symptom
}

This article was published in the following Dove Press journal:

Risk Management and Healthcare Policy

\section{Ping-Xin $\mathrm{Qi}^{1,2, *}$ \\ Jia-Ni Zou ${ }^{3, *}$ \\ Jian Zhu (iD ${ }^{2}$ \\ Bin Huang ${ }^{4}$ \\ Er-Ping $\mathrm{Xi}^{2}$ \\ Xu-Hui Gao ${ }^{2}$}

'School of Medicine, Wuhan University of Science and Technology, Wuhan 430065, People's Republic of China; ${ }^{2}$ Department of Thoracic Cardiovascular Surgery, General Hospital of Central Theater Command of the People's Liberation Army, Wuhan 430070, People's Republic of China; ${ }^{3}$ Department of Radiology, General Hospital of Central Theater Command of the People's Liberation Army, Wuhan 430070, People's Republic of China; ${ }^{4}$ The First School of Clinical Medicine, Southern Medical University, Guangzhou 510515, People's Republic of China

*These authors contributed equally to this work
Correspondence: Xu-Hui Gao; Er-Ping Xi Department of Thoracic Cardiovascular Surgery, General Hospital of Central Theater Command of the People's Liberation Army, 627\#, Wuluo Road, Wuchang District, Wuhan, Hubei 430070,

People's Republic of China

Tel +86- | $3307 \mid 95757$

Fax +86-27-50772388

Email whzyygxh@I26.com;

wo535I@I26.com

\begin{abstract}
A 55-year-old man was treated at the village hospital with six months medical history of recurrent chills and fever. Due to the lack of imaging examination, antipyretic and anti-infective medications were given. Although symptomatic treatment can relieve fever symptoms, symptoms easily flare up again two to three days after taking the drug. Later, the patient suffered from fever again during the COVID-19 epidemic and was sent to our hospital for isolation and treatment. During this hospitalization, chest CT examination is mandatory for all patients in order to meet the requirements of epidemic prevention and control. This led to the inadvertent discovery of a large cystic solid mass in the right thoracic cavity communicating with the esophageal lumen. The patient was preliminarily diagnosed as giant midesophageal diverticulum after three-dimensional CT image reconstruction of the chest was reviewed. Considering the patient's persistent fever with poor nutritional status, we decided to temporarily place two gastric tubes (diverticulum decompression and gastrointestinal nutrition), and antibiotics were used at the same time as another main treatment. However, after the symptoms eased and nutritional status improved, he refused all further treatment. We believe that this patient's diverticulum is very classic, and the treatment plan is highly integrated with the needs of epidemic prevention and control and achieves a satisfactory therapeutic effect, so we hope to provide colleagues with new diagnosis and treatment enlightenment through this case.
\end{abstract}

Keywords: giant midesophageal diverticulum, symptom, treatment, imaging

\section{Introduction}

Esophageal diverticulum is very rare gastrointestinal disease. According to the location of the diverticulum, it can be divided into pharyngeal diverticulum, middle esophageal diverticulum, and distal diverticulum (epiphrenic). ${ }^{1}$ Midesophageal diverticulum is defined as $5 \mathrm{~cm}$ above and below the level of the trachea bifurcation. Midesophageal diverticulum is usually asymptomatic, with rare clinical manifestations of dysphagia, reflux and chest pain. ${ }^{2}$ We herein report the case of a patient with a giant mid-esophageal diverticulum with fever as the only symptom. Since this case is very classic, not only the diverticulum is very large, but also a good medical teaching effect can be obtained through the three-dimensional computed tomography imaging for diagnosis.

\section{Case Report}

A 55-year-old man went to a village hospital for recurrent chills and fever without any other discomfort. This fever (highest $39^{\circ} \mathrm{C}$ ) with chilly intermittently for 6 months. There was no significant family history or previously infectious disease such as pulmonary tuberculosis, mediastinitis, hilar lymphadenitis and tumors. Physical examination disclosed that breath sounds of both lungs were thick, and there are obvious wet rales in the 
right lung, cardiac rhythm and heart sound were normal. The results of laboratory examination indicated that neutrophil count, erythrocyte sedimentation rate and C-reactive protein were all higher than normal values. No obvious abnormalities were found in the laboratory indexes of tumors. Due to limited medical conditions in primary hospitals, fever symptoms in patients are often thought to be caused by common influenza or upper respiratory tract infection (URTI). Coincidentally, the cephalosporin anti-infective treatment was effective for his symptoms. However, symptoms were easy to flare up again two to three days after taking the drug. Later, the patient suffered from fever again during the COVID-19 epidemic and was sent to our hospital for isolation and treatment. Timely nucleic acid testing of SARS-CoV-2 was negative. Computed tomography scan of the chest showed a $7 \mathrm{~cm}$ large pouch of the right esophageal wall below the level of the carina, filled with retained food, demonstrating giant midesophageal diverticula (Figure 1). Due to concerns about the risk of perforation caused by persistent infection within the giant diverticulum, upper gastrointestinal imaging and gastrointestinal decompression within the diverticulum were performed simultaneously (Figure 2). Through the placement of two gastric tubes (one was inserted into the diverticulum for suction residual chymus, the other was inserted into the stomach for supplying nutrition), we were able to provide good enteral nutritional support without causing residue accumulation within the diverticulum. After the patient's esophageal diverticulum was emptied by the gastric tube, we decided to perform a esophagogastroduodenoscopy examination to determine whether the patient had esophageal diverticulum canceration. This examination revealed a diverticulum with a wide orifice which was located $30 \mathrm{~cm}$ from the incisor had no signs of inflammation, ulceration (Figure 3). After fasting, closed drainage, antibiotic therapy, and nutritional support for a week, the patient's symptoms completely disappeared. However, he refused all further invasive therapy and admitted to the hospital regularly for review.

\section{Discussion}

Esophageal diverticulum is a rare disease of the gastrointestinal system. Acquired esophageal diverticulum classified according to mechanisms of formation as pulsion or traction diverticulum, they may occur in the pharyngoesophageal transition area, midesophagus or distally (epiphrenic). Midesophageal diverticulum is traditionally considered traction diverticula secondary to mediastinal inflammatory reaction such as pulmonary tuberculosis, although anthracosis, histoplasmosis and treated non-Hodgkin's lymphoma have also been reported. Because of the pulmonary tuberculosis, which is one of the primary causes of traction diverticulum, is in decline in the western world, recent studies have shown that midesophageal diverticulum is most often pulsion in nature. ${ }^{1,2}$ Common symptoms of esophageal diverticulum include dysphagia, regurgitation, chest pain, and aspiration. However, many patients with esophageal diverticulum presented no symptoms, which causes most esophageal diverticulum to be found incidentally. ${ }^{3}$ Although esophageal radiography with an oral contrast agent is the most efficient diagnostic method for this disease, other investigations such as chest computed tomography is helpful in evaluating atypical symptoms or findings.
A

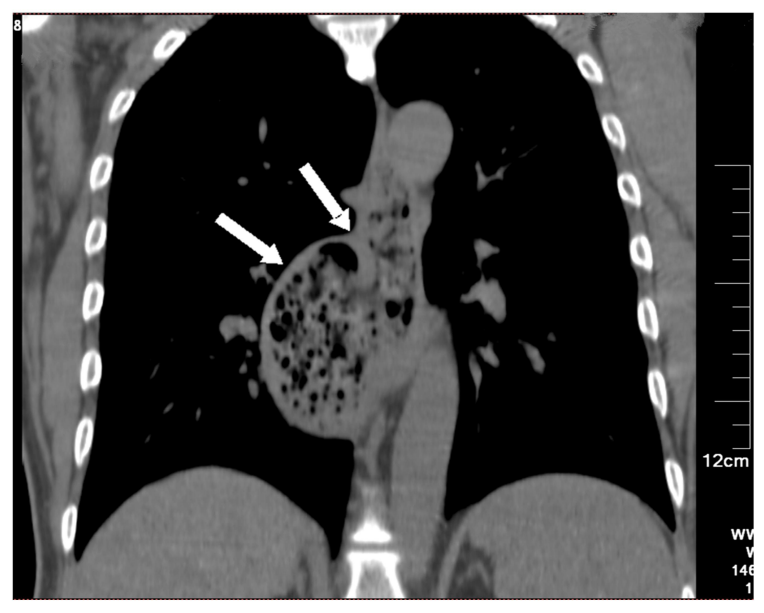

B

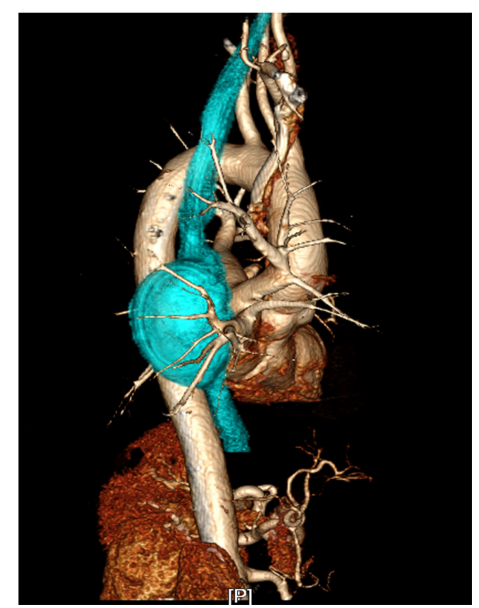

Figure I (A) Mediastinal window of CT scan frontal plane shows that outpouching from the esophagus contains a giant cystic solid "mass" (Indicated by the arrows) under the tracheal bifurcation. (B) Three-dimensional CT scan clearly reveal the position and the size of the "mass", and the relationship between "mass" and surrounding internal organs. 


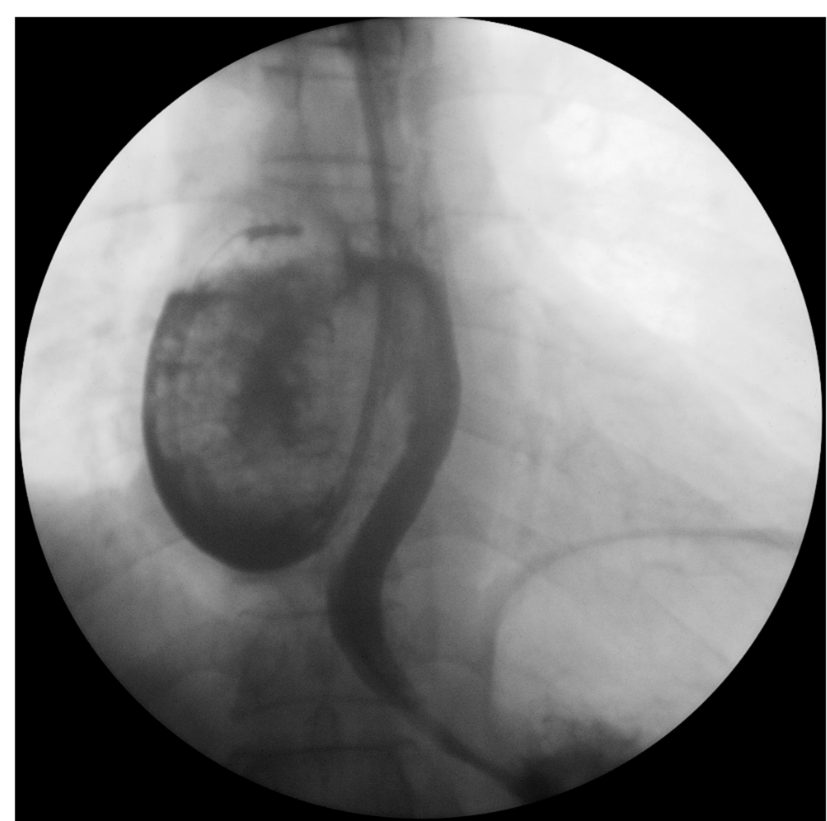

Figure 2 Esophagogram demonstrated the "mass" is a giant esophageal diverticulum filled with food debris, no evidence of contrast medium leakage, and showed the distal esophagus was unobstructed without contrast agent stasis, and the mucosa and structure at the gastroesophageal junction were normal. Two nasogastric tubes were inserted (one was inserted into the esophagus for suction residual chymus, the other was inserted into the stomach for supplying nutrition).

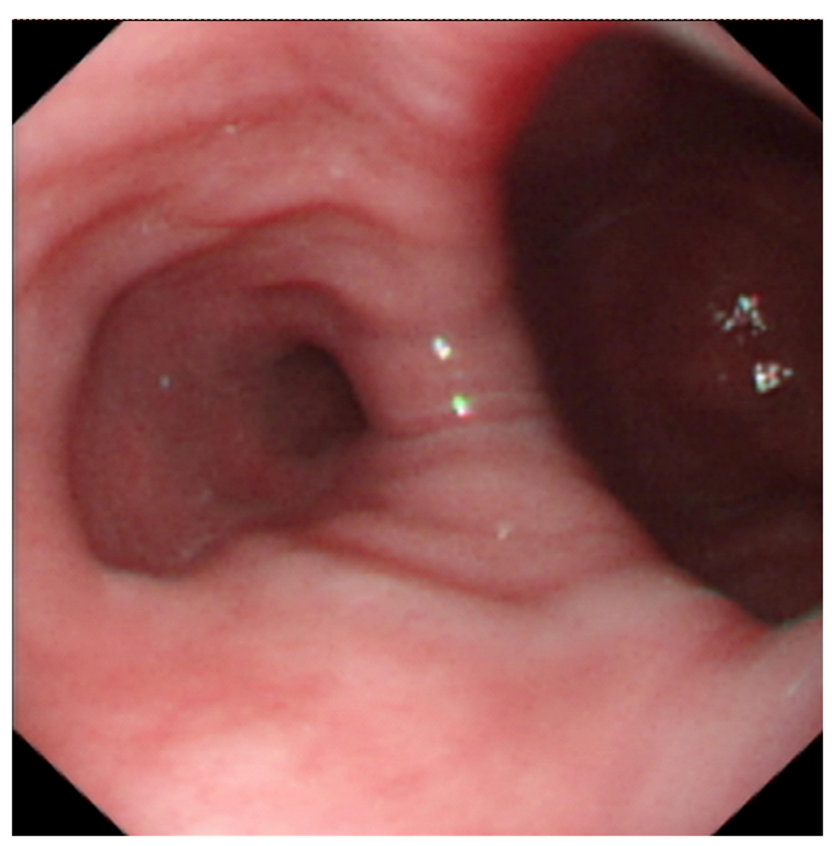

Figure 3 A distant view of upper Gl endoscopy confirmed a giant diverticulum with a wide orifice and the esophageal lumen, and showed a diverticulum mucosa is normal, without inflammation or ulcers.

Computed tomography could define a mass lesion arising from a perforated or malignant diverticulum, it is intuitively showing the location, size, shape of the esophageal diverticulum and its relationship with the chest trachea and the great blood vessels of the heart. Barium esophagram and other upper gastrointestinal radiography can only confirm the diagnosis of esophageal diverticulum, but the mucosal lesions in the esophagus and diverticulum cavity are not clearly diagnosed. ${ }^{4}$ Chronic irritation by food residues, repeated inflammation of the cavity wall, and periodic damage and repair of the luminal mucosa may induce malignant lesions. ${ }^{5}$ So, endoscopy is recommended irrespective of radiological appearances, it is essential for identifying esophageal tumors or strictures as causes of dysphagia, and in differentiating radiographic irregularities while permitting biopsies for histology. For symptomatic patients, surgery should be reserved. This is a consensus. The greatest risk of morbidity and mortality caused by non-surgical intervention is due to aspiration. Therefore, surgical intervention is mandatory when there is a risk of aspiration, even in the absence of esophageal symptoms. ${ }^{6}$ Although midesophageal traction diverticula are usually small and do not require surgery, resection is the option in patients with large midesophageal diverticulum via thoracoscopy or thoracotomy such as diverticulectomy or additional esophageal myotomy. ${ }^{7}$ In addition to traditional surgical treatment, minimally invasive endoscopic approaches have gained popularity in recent years. Endoscopic therapy usually includes endoscopic diverticulectomy, endoscopic submucosal dissection using a variety of special equipment, and diverticular peroral endoscopic myotomy. This allows patients to have more options for the treatment of the disease. The common complications of traditional surgical treatment such as perioperative aspiration, postoperative leaks and postoperative pneumonia should be fully considered. Although endoscopic approaches are regarded as lower complication rates and equivalent efficacy to conventional surgery, intraoperative esophageal perforation is one of the most frightening complications, which will lead to failure of endoscopic treatment or even immediate transfer to thoracotomy to save the patient's life. Treatment of a giant mid-esophageal diverticulum with endoscope is a difficult procedure and requires technical expertise. Regardless of the surgical option, the pros and cons should be carefully weighed, especially during the epidemic of COVID-19. ${ }^{8}$

Unlike previous reports of esophageal diverticulum, our patient's chief complaint was fever rather than dysphagia or regurgitation. Unfortunately, many diseases can cause chills and fever, which inevitably leads doctors at primary hospitals to misdiagnose the disease. When the esophagus collapses, traction diverticula tend to empty, which further increases the 
difficulty of diagnosing the disease through imaging. Fortunately, because of fever, he was highly suspected of being COVID-19 and was diagnosed as esophageal diverticulum when isolated in a larger hospital.

In our case, the three-dimensional CT of the chest shows a classic image of a giant middle esophageal diverticulum, which clearly shows the position and the size of the diverticulum, and the relationship between diverticulum and surrounding internal organs. In addition, the treatment of our case is quite unique, using two gastric tubes (one was inserted into the esophagus for suction residual chymus, the other was inserted into the stomach for supplying nutrition) palliated patient's symptoms, which improved the quality of life, and got a satisfactory preliminary status. Moreover, it is reasonable to effectively and safely stop nonurgent and nonemergency surgery during the COVID-19 epidemic. ${ }^{9,10}$ This kind of treatment is very suitable and effective for the actual situation. In short, we feel that our case has distinctive images, simplified treatment methods and accurate treatment effects, which can help patients relieve their pain and suffering, and can give doctors in basic hospitals some enlightenment on the treatment of related esophageal diseases. It can also provide medical students with a classic image case of esophageal diverticulum.

\section{Ethics}

This research complies with the guidelines for human studies and is in accordance with the Declaration of Helsinki. The ethics Review Committee of General Hospital of Central Theater Command of the People's Liberation Army approved the use of clinical data of this patient in this study ([2020]035-1).

\section{Informed Consent for Publication}

Written informed consent was obtained from the patient for the publication. The patient provided written informed consent to participate in this study.

\section{Acknowledgment}

These authors contributed equally to this work and should be considered as co-first authors: Ping-Xin Qi and Jia-Ni Zou.

\section{Funding}

This research did not receive any specific grant from funding agencies in the public, commercial, or not-forprofit sectors.

\section{Disclosure}

The authors declare that they have no conflicts of interest for this work.

\section{References}

1. Thomas ML, Anthony AA, Fosh BG, Finch JG, Maddern GJ. Oesophageal diverticula. Br J Surg. 2001;88:629-642. doi:10.1046/ j.1365-2168.2001.01733.x

2. Sonbare DJ. Pulsion diverticulum of the oesophagus: more than just an out pouch. Indian J Surg. 2013;12:955.

3. Smith CD, et al. Esophageal strictures and diverticula. Surg Clin North Am. 2015;95:669-681. doi:10.1016/j.suc.2015.02.017

4. Renato S, Fernando A, Prachand VN, et al. Epiphrenic diverticulum of the esophagus from pathophysiology to treatment. $J$ Gastrointest Surg. 2010;14:2009-2015. doi:10.1007/s11605-010-1216-9

5. Herbella FA, Patti MG, Patti MG, et al. Esophageal diverticula and cancer. Dis Esophagus. 2012;25:153-158. doi:10.1111/j.14422050.2011.01226.x

6. Herbella FA, Patti MG. Modern pathophysiology and treatment of esophageal diverticula. Langenbecks Arch Surg. 2012;397:29-35. doi:10.1007/s00423-011-0843-2

7. Zaninotto G, Portale G, Costantini M, et al. Therapeutic strategies for epiphrenic diverticula: systematic review. World $J$ Surg. 2011;35:1447-1453. doi:10.1007/s00268-011-1065-z

8. Sato H, Takeuchi M, Hashimoto S, et al. Esophageal diverticulum: new perspectives in the era of minimally invasive endoscopic treatment. World J Gastroenterol. 2019;25:1457-1464. doi:10.3748/ wjg.v25.i12.1457

9. Doglietto F, Vezzoli M, Gheza F, et al. Factors associated with surgical mortality and complications among patients with and without coronavirus disease 2019 (COVID-19) in Italy. JAMA Surg. 2020;155 (8):691-702. doi:10.1001/jamasurg.2020.2713

10. Nassar AH, Zern NK, McIntyre LK, et al. Emergency restructuring of a general surgery residency program during the coronavirus disease 2019 pandemic: the University of Washington experience. JAMA Surg. 2020;155:624-627. doi:10.1001/jamasurg.2020.1219
Risk Management and Healthcare Policy

\section{Publish your work in this journal}

Risk Management and Healthcare Policy is an international, peerreviewed, open access journal focusing on all aspects of public health, policy, and preventative measures to promote good health and improve morbidity and mortality in the population. The journal welcomes submitted papers covering original research, basic science, clinical \& epidemiological studies, reviews and evaluations, guidelines, expert opinion and commentary, case reports and extended reports. The manuscript management system is completely online and includes a very quick and fair peer-review system, which is all easy to use. Visit http://www.dovepress.com/testimonials.php to read real quotes from published authors. 